\title{
Carnets
}

Revue électronique d'études françaises de l'APEF

Première Série - 2 Numéro Spécial 10-11 | 2011

D'un Nobel l'autre

\section{Réflections préliminaires à une étude du thème de l'eau et de l'extase dans la littérature et le cinéma franco-vietnamiens}

\section{Géorges van den Abbeele}

\section{OpenEdition}

Journals

Édition électronique

URL : http://journals.openedition.org/carnets/5728

DOI : $10.4000 /$ carnets. 5728

ISSN : 1646-7698

Éditeur

APEF

Édition imprimée

Date de publication : 1 janvier 2011

Pagination : 215-243

Référence électronique

Géorges van den Abbeele, « Réflections préliminaires à une étude du thème de l'eau et de l'extase dans la littérature et le cinéma franco-vietnamiens », Carnets [En ligne], Première Série - 2 Numéro Spécial 10-11 | 2011, mis en ligne le 16 juin 2018, consulté le 19 avril 2019. URL : http:// journals.openedition.org/carnets/5728; DOI : 10.4000/carnets.5728

\section{(2) $(1) \Theta$}

Carnets est mis à disposition selon les termes de la licence Creative Commons - Atribution - Pas d'utilisation commerciale 4.0 International. 


\title{
RÉFLECTIONS PRÉLIMINAIRES À UNE ÉTUDE DU THĖME DE L’EAU ET DE L'EXTASE DANS LA LITTÉRATURE ET LE CINÉMA VIETNAMIENS
}

\author{
Georges VAn Den AbBeEle \\ Northeastern University, Boston \\ g.vandenabbeele@neu.edu
}

\begin{abstract}
Résumé
Le thématique de l'eau dans la littérature et le cinéma franco-vietnamiens semble désigner un lien profond et intime entre le corps et le "lieu" vietnamiens (il ne s'agit évidemment pas des poncifs occidentaux de terre et de sol). Condensée par Nguyên Du dans la scène célèbre du Truyên Kiêu, ce texte fondamental à la culture du Viêt-nam, l'image du corps féminin dans son extase aquatique dépasse toute captation simplement voyeuriste ou vulgairement lubrique pour nous tracer la voie "sans traces" du chemin fluvial qui remonte vers l'âme profonde (comme on dit "pays profond") du dât nuòc. II s'agit donc d'un topos à lire dans sa spécificité culturelle, au delà de toute idéalisation de "sang et sol", vers une expression on ne peut plus lyrique d'identité localisée, une affirmation du chez soi collectif et donc d'une résistance limpide mais inlassable à toute imposition de l'extérieur.
\end{abstract}

\begin{abstract}
The thematics of water in Franco-Vietnamese literature and film describes a deep and intimate link between the body and place in Viet Nam (without in any way referencing Western stereotypes of place and ground). As represented by Nguyên Du in a famous episode from the Truyên Kiêu, that key text of Vietnamese culture, the image of the feminine body in an aquatic ecstasy rejects any simply voyeuristic or vulgarly lascivious reception to trace the "traceless" path of the fluid route that leads back up to the inner heart (or "heartland") of the dât nuòc. The topos is to be read in its cultural specificity, far beyond any idealization of "blood and soil," as the lyrical expression of localized identity, an affirmation of collective being together and, hence, of a limpid but indefatigable resistance to any external impositions.
\end{abstract}

Mots-clés: Viet-nam, littérature et cinéma, poétique de lieu, corps féminin

Keywords: Viet Nam, literature and film, poetics of place, feminine body 
Vers le milieu d'Une histoire d'amour (1995), film que sa réalisatrice Trinh T. Minh-ha présente comme une "traduction" libre, sous forme de "haïku", du célèbre poème de Nguyên Du, le personnage de Kiêu rappelle quelques moments où la conduite autrement vertueuse de son homonyme littéraire s'avère plutôt blâmable:

Par exemple, Kiêu ose visiter son Romeo, Kim Truong, la nuit chez lui - démarche ultra-extraordinaire pour une femme aussi bien élevée. Puis, le poète nous régale d'une scène érotique où un de ses amants l'entrevoie se baignant nue au clair de la lune. Enfin et surtout, Kiêu reste, malgré le pathos de sa vie, une créature dont les pulsions de la passion l'amènent à aimer non pas un seul, mais trois hommes - avec un amour différent pour chacun. (Minh-ha, 1999: 18).

On voit bien que ce sont ces quelques actes de transgression que Trinh Minh-ha met en relief afin de démentir une interprétation traditionnelle de Kiêu qui insisterait sur les sacrifices que demande sa fidélité confucéenne à la famille aussi bien que les vertus de sa passivité bouddhiste à la loi du Karma. Pour Trinh Minh-ha, loin d'être la simple victime des circonstances maléfiques qui s'accumulent entre un destin néfaste et une société impitoyablement patriarcale, Kiêu montre une volonté et un esprit d'autonomie, qui, certes, restent passablement circonscrits par les forces du destin et de l'ordre social (seul, l'Occident semble imaginer que l'expression d'un tel esprit suffit pour réaliser le fantasme d'être "maître de son destin"). Au contraire, le génie de Nguyên Du est d'avoir su, par un syncrétisme on ne peut plus vietnamien, intégrer une tradition indigène de femmes fortes (qui remonte aux sœurs Truong) avec les importations religieuses de la Chine (Confucius) et de l'Inde (Bouddha). Le scandale du Kiêu qui est aussi à l'origine de son succès littéraire est d'avoir su mettre en scène un modèle de persévérance humaine (féminine en l'occurrence) qui donne de l'espoir contre tous les obstacles que la nature ou la société puissent élever contre nous. Cela, c'est encore le message explicite des vers ultimes du poème: "Et si un lourd Karma pèse sur notre destin, ne récriminons pas contre le ciel et ne l'accusons pas d'injustice. La racine du bien réside en nous-mêmes. Cultivons cette bonté du cœur qui vaut bien plus que le talent." (Du: 173, v. 3249-3252; c'est moi qui souligne).

Revenons à la scène de la baignade en tant qu'une des transgressions "exemplaires" de Kiêu. A vrai dire, dans cette transgression, s'il faut l'appeler ainsi, il ne s'agit pas vraiment d'une action directe de sa part comme dans les deux autres, mais comme nous allons voir, c'est son être même qui se trouve en jeu. Voici la scène telle qu'elle paraît dans la traduction française de Kim-Vân-Kiêu réalisée par Xuân Phuc et Xuân Viêt: 
Cependant, sous la lune, les râles d'eau lançaient leurs appels à l'été. Par-dessus les murs scintillaient les flammes des grenadiers en fleur. Dans la chambre tendue de gaze, par une belle heure de loisir, elle fit tomber les rideaux roses pour baigner son corps de fleurs dans une décoction d'orchidées. Idéale structure, ce temple d'amour, façonné par les dieux, se dresse en sa blancheur d'ivoire, en sa pureté de jade. Le jeune homme, émerveillé, ne put cacher son admiration. (Du: 93, v. 1307-1313; traduction modifiée)

Le rideau rose est-il tombé par mégarde, suscitant ainsi l'œil intrusif du voyeur? Ou bien, est-ce par dessein afin de provoquer le jeune homme, Thuc, par un spectacle qui expose la beauté divine ("façonné par les dieux") du corps féminin? Le texte, comme il l'est souvent le cas dans le poème de Nguyên Du, reste bien ambigu en ce qui concerne les intentions de Kiêu quelles qu'elles soient, louables ou bien blâmables.

Que ce soit transgression volontaire ou pas, ce que dévoile cette baignade c'est Kiêu dans l'intimité de son être, dans le plaisir d'une autonomie privée et tout à fait à l'intérieur où elle ne dépend de personne, un espace sacré on ne peut plus loin des déceptions et des compromis auxquels elle doit se livrer journellement, ne fût-ce que pour survivre. Mais cette arrière-boutique personnelle n'est pas qu'un simple refuge, car l'endroit où son corps se révèle dans toute sa splendeur architecturale ("idéale structure, ce temple d'amour"), où elle se livre à l'extase de se baigner dans une eau parfumée de fleurs, ce lieu de recueillement est là aussi où en se ruisselant jusqu'à devenir liquide, elle recèle, selon le poème, une force redoutable, un "étrange pouvoir de la beauté dont la vague dévastatrice renverse sur son passage comme en se jouant, maisons et palais" (v. 1301-02). Cette vision de la femme marée haute, voire "tsunami" prévoit, certes, le moment bien ultérieur où Kiêu, devenu le compagnon du grand guerrier Tù-Hai, prend sa vengeance, exacte et sanglante, sur les divers personnages qui lui ont fait mal au cours de ses aventures. (Bien que Trinh ne la mentionne pas parmi les transgressions de Kiêu, cette scène n'a pas manqué aussi de soulever maintes discussions sur la conduite parfois moins que vertueuse de l'héroïne.) D'autre part, ce pouvoir féminin qui dans le contexte rappelle aisément les grands exploits des sœurs Truong et d'autres guerrières vietnamiennes dans la lutte contre la domination chinoise, démarque un au-delà de la maîtrise humaine, voire masculine, qui reste malgré tout un attrait irrésistible pour les hommes, tel le jeune Thuc qui tombe éperdument amoureux de Kiêu, si bien qu'il lui écrit "un poème de forme classique [Duòng]". Malgré ce grand effort lyrique, Kiêu ne lui donne pas l'accueil qu'il cherchait:

Les sentiments que vous exprimez me touchent profondément. Ce ne sont que paroles de perle et de jade, ce ne sont que sentences de brocart. Selon mes faibles moyens, j'aurais dû vous donner la réplique en improvisant les mêmes rimes. Mais la 
nostalgie dont je souffre m'en empêche. Mon cœur est là-bas avec les nuages dorés du pays natal. Quant au poème en réponse, je vous demande donc grâce pour aujourd'hui. (v. 1315-20).

L'exilée Kiêu ne peut (ou ne veut) pas répondre aux désirs de Thuc, parce que son "cœur" reste dans son pays natal, "sous les nuages d'or" selon un proverbe traditionnel chinois. La baignade au clair de lune est donc aussi une sorte de retour à la patrie pluvieuse, une relève rituelle et privée de la souffrance de l'exil Comme nous le rappelle Kim Lefèvre, "dans la langue vietnamienne pays s'écrit 'Dât-Nuóc', mot qui signifie 'Terre et Eau."' (Lefèvre, 1990: 149). L'expression prend tout son sens dans la topographie du Viêt-nam, pays coincé entre les hautes montagnes de la cordillère annamite qui s'élève à plus que trois mille mètres de hauteur et une côte longue d'environ trois mille kilomètres, entre lesquelles se frayent les cours d'à peu près un millier de fleuves, y compris les grands systèmes riverains: le Rouge, des Parfums, le Mékong, ou pour les appeler par leurs noms vietnamiens, sông Hông, sông Huong, Cuu Long. L'eau de la mer condensée en montagne laisse tomber d'énormes pluies (les moussons) qui gonflent les eaux tumultueuses des rivières. Climat idéal pour la culture du riz, nourriture aussi fondamentale et symboliquement féconde que ne l'est le pain chez les Occidentaux, si bien que la rizière, ou lúa nuóc, représente bien le pays profond et profondément aqueux du dât nuóc. Allégorie du pays, le corps ruisselant de Kiêu se baignant affirme une identité vietnamienne que l'on garde à fond et qu'aucun pouvoir proxénète, colonialiste, impérialiste ou totalitaire ne pourra jamais arriver à "déterrer." Loin de se faire approprier par un quelconque regard de l'Autre, l'extase de l'eau nous laisse entrevoir la source profonde d'une résistance efficace et imbattable, une résistance à la sóng khuynh thành.

La scène de la baignade de Kiêu se reproduit textuellement dans le film de Trinh Minh-ha. On entend chanter le texte du Kiêu en voix "off" tandis qu'on voit le dos d'une femme qui se baigne en pleine nuit. Elle remplit tasse après tasse d'eau qu'elle laisse alors couler sur son corps. Son plaisir est évident, mais le plan suivant, en changeant d'angle de prise, nous laisse entrevoir aussi la silhouette d'une femme (sans doute la tante, chez qui habite Kiêu) qui regarde avec intérêt cette femme, que nous reconnaissons maintenant être bien le personnage principal de Kiêu elle-même, bien que celle-ci semble ignorer tout à fait qu'on la regarde. En remplaçant le regard du jeune homme par celui de la vieille, Trinh Minhha semble insister davantage sur l'autonomie féminine dans une intimité qui ne dépend d'aucun homme.

Ce même plaisir se voit aussi au moment où Kiêu se retire de l'appartement de son ami photographe, Alikan, au moment où elle l'a surpris en train de prendre des photos pornos d'une jeune blanche étalée cuisses ouvertes au dos d'une motocyclette. Visiblement 
gênée par ce qu'elle vient de voir, Kiêu remonte à l'extérieur, claque la porte derrière elle et traîne un peu sur le seuil, tandis qu'il commence à pleuvoir. On entend encore chanter des vers du Kiêu sur la douleur du lot des femmes. Lentement, elle se rend compte de la pluie, et comme si elle se retrouvait, elle se plaît à se faire mouiller par les gouttes qui tombent. On passe à un nouveau plan, cette fois du point de vue subjectif, scène de la mémoire de Kiêu où nous la voyons comme petite fille jouant au bord de l'eau, plan dont la nostalgie évidente pour la terre-eau du Viêt-nam se trouve encore brusquement interrompue par la voix de son autre ami, Minh (nom qui veut dire "sagesse" en Vietnamien) qui l'appelle par son nom. Comme réponse, elle lui explique que 'Iorsqu'il pleut, l'odeur de la terre et de l'herbe [lui] donne toujours de la nostalgie de chez [elle]." II la ridiculise, puis il lui propose de monter sur son vélo -- version innocentée du motard pervers? -- avant qu'elle ne se trouve, dit-il, "toute mouillée." Elle accepte son offre sans enthousiasme, puis commence à s'amuser quand même en redirigeant son attention vers la pluie qui tombe sur elle et son copain, jusqu'à ce qu'ils commencent à rire ensemble tous les deux.

Passe au plan suivant qui nous montre Kiêu s'éveillant seule dans son lit. On entend toujours la même sonorité de la pluie qui tombe, et Kiêu qui sort de l'abri pour mieux l'éprouver. Voici les indications scénographiques que donne Trinh Minh-ha: "She enjoys getting wet. With both hands, she spreads the water on her face and neck, making her nightshirt cling to her body. We SEE her absorbed in feeling the rain falling and wetting her chest. She sensually spreads the water on her arms and chest." (Minh-ha, 1999: 114). Ici, il n'y plus de spectateur en scène et le plaisir extatique de Kiêu est plus qu'évident dans la mesure où il ne s'agit que d'un plaisir privé et personnel pour elle seule, où elle se retire et se réaffirme sans ambages. Dans la scène suivante, elle refusera définitivement de poser nue pour le photographe, Alikan, et poursuit avec de plus en plus d'acharnement ses enquêtes du Kiêu et du destin de son homonyme en tant que modèle de résistance.

Trinh Minh-ha est loin d'être le seul interprète contemporain de cet épisode du Kiêu. Un traitement différent se voit dans le film de Tran Anh Hung, L'odeur de la papaye verte (1993). On nous y présente la vie d'une jeune servante réfugiée qui travaille pour une famille saïgonnaise. Arrivée à l'âge adulte, "Mui" est congédiée et passe dans la maison de Khuyen, jeune compositeur riche et très francisé qui passe ses jours à jouer du Chopin au piano et les soirs à sortir avec sa fiancée bien bourgeoise. On voit Mui en train de préparer les repas, à nettoyer et à coudre, etc. Puis, dans la scène la plus célèbre du film, on la voit, à travers une série de coupes multiples en faux raccord, prendre un bain nocturne, tout en prenant plaisir dans ce moment d'intimité personnel, dans cette "heure de loisir", où elle n'aura plus à répondre aux besoins du maître. Aucune indication directe que l'on observe effectuant cette reprise du Kieu, mais dans les scènes suivantes on voit le jeune homme s'ennuyer de plus en plus de sa fiancée pour prendre un intérêt de plus en plus accru pour sa servante. Cet 
intérêt s'expose concrètement quand il compare un vieux buste indochinois avec la figure de la belle Mui, et dessine son portrait d'après le buste de façon à renforcer cette identité entre la figure de Mui et celle d'un Viêt-nam traditionnel. D'un côté, le désir qu'il éprouve pour sa servante ne peut sembler qu'une projection esthétique, voire esthète, annoncée déjà par la scène de la baignade, qui présente Mui pour la première fois dans le film comme être belle et désirable. Mais en même temps, la métaphore architecturale qui décrit la beauté de Kiêu en tant que temple idéal"façonné par les dieux" nous prépare aussi pour l'association de la beauté de Mui avec le buste pierreux et ancien d'une divinité de temple. D'autre part, cet éveil esthétique à la beauté de Mui est aussi pour Khuyen un rappel à ses origines vietnamiennes, représentées par Mui/Kiêu. Se sentant de plus en plus négligée par Khuyen, sa fiancée sort de la maison pour se retrouver sous une pluie ardue. Ne pouvant réprimer sa curiosité, elle retourne regarder par la fenêtre son fiancé qui joue au piano tandis que Mui fait ses affaires, vision qui propose l'image d'une joie domestique dont elle est dorénavant exclue. On la voit donc qui se retourne devant nous et se met sous la drache à pleurer sans consolation. La scène propose donc une inversion structurale de la baignade de Kiêu: c'est maintenant la femme trempée dans l'eau qui se trouve à l'extérieur et qui, sans qu'on la voie, regarde à l'intérieur l'homme (et la rivale) bien à l'abri. A noter aussi que pour la femme occidentalisée, l'eau tombante n'est plus source de confort et de plaisir mais image pathétique ou état d'âme de la tristesse, comme si la référence littéraire n'était plus le poème national vietnamien mais le symbolisme de Paul Verlaine: "Il pleure dans mon cœur comme il pleut sur la ville" (Romances sans paroles).

Par contre l'image vietnamienne de l'eau extatique est reprise dans la scène suivante où l'on voit encore Mui se baignant la nuit (toujours avec le même style de découpage en faux raccord), séquence qui précède la grande scène où Khuyen ose enfin entrer dans la chambre de Mui. Devenus amants, Khuyen apprend donc à lire a Mui, qui dans la scène ultime du film lit un poème vietnamien qui reprend encore une fois le thème de l'eau en tant que "source" de l'identité:

L'eau de la source, blottie dans un trou de roche, miroite doucement quand on la remue. Les tremblements du sol ont donné naissance à de fortes vagues qui s'abattent dans une houle irrégulière, à la surface, sans déborder. S'il y a un verbe qui veut dire, "se mettre en mouvement harmonieusement," il faudrait l'employer ici. Les cerisiers, pris dans les ténèbres, s'étendent et se rebroussent, se balancent et se tordent, toujours au rythme de l'eau. Mais l'intéressant c'est: quoi qu'ils changent, ils gardent toujours la forme d'un cerisier. 
L'affirmation d'une identité vietnamienne à travers le thème de l'eau s'affiche bien évidemment aussi dans le récit de l'écrivaine eurasienne, Kim Lefèvre, Retour à la saison des pluies (Paris: Aube, 1995), dont le titre déjà nous invite à faire cette association. Pour Lefèvre, non seulement le retour à son pays natal se décrit-il comme une remontée d'eau: "comme un long fleuve dont l'amont serait si éloigné qu'il ne paraît à présent enveloppé de brume" (p. 131). Sa vie, dit-elle, "menée ailleurs que sur le sol vietnamien" se définit comme un séjour à l'étranger, "flottant à des milles de l'endroit où j'ai pris ma source" (p. 13). Plus profondément, le souvenir du Viêt-nam de son enfance, quand il s'avère positif (et non celui négatif, des malheurs soufferts par elle en tant qu'enfante eurasienne ou "métèque blanche"), ce souvenir positif, dis-je, tend à s'identifier avec l'expérience de l'eau et de la pluie. Déjà dans Métèque blanche (1989), elle nous avait fait part de son souvenir le plus primordial et aussi le plus réconfortant:

C'était une nuit d'automne à Hanoi. Je me souviens de la fraîcheur pénétrante du crachin - cette petite pluie fine tonkinoise --, de la douce sensation du dos nu de la nourrice, de sa peau tout à la fois flasque et élastique. Accrochée à sa chair comme à un hamac chaud et soyeux, je savourais le bonheur de la route sans fin. J'eusse aimé que ma vie entière fût ce voyage sans destination, suspendue à la peau chaleureuse de ma nourrice, tandis que la mienne s'offrait avec gratitude au crachin tonkinois. (15)

Et voici, comme elle décrit son débarquement à l'aéroport de Saigon presque cinquante années plus tard:

Un dernier coup d'œil à la foule en attente et me voici à l'air libre. II pleut, c'est la saison des moussons. Une pluie lourde, violente. Je repense à d'autres pluies semblables, lorsque enfant je me précipitais joyeusement sous les trombes d'eau qui dégringolaient du toit. En quelques instants la rue s'est transformée en rivière charriant des bulles à la dérive et la grande personne que je suis aujourd'hui regarde avec nostalgie cet écoulement d'eau trouble en frissonnant. (Lefèvre, 1995: 139-40)

Si l'eau, et surtout l'eau sous forme de pluie, la ramène à une enfance vietnamienne perdue, il ne faut pas non plus y imposer des images occidentales et prudes d'une supposée innocence enfantine. Car l'enfance retrouvée dans l'expérience du corps mouillé propose non seulement l'attachement au sol vietnamien (ou plutôt, devrait-on dire, sol et eau vietnamiens: dât nuòc viêt nam) mais une continuité sans gêne et sans contradiction aucune avec l'extase corporel. Voici comme Kim Lefèvre décrit sa sœur Oanh, non pas métèque eurasienne comme elle mais "pleinement" vietnamienne, belle à susciter de l'inquiétude chez sa mère: "Ma mère croyait qu'à l'instar de Kiêu, la beauté de sa fille ne pouvait lui apporter 
qu'une vie de malheurs et de souffrances" (p. 102). La mère se soulage dès que Oanh perd pourtant de sa beauté tout en agrandissant un peu, mais voici l'image érotisée de Kiêu baignant qui revient tandis que la narratrice dénombre les "plaisirs simples" qui occupent sa sœur tels "se dénuder sous l'averse" ou celui-ci: "Elle passait des heures à verser de l'eau fraîche sur la peau moite, les paupières closes, tout entière absorbée par ses sensations. Puis, un jour, je la vis serrer étroitement entre les cuisses un oreiller tandis que sur sa figure s'irradiait une volupté extatique" (p. 104).

Corps privé de la femme qui s'adonne à son plaisir, corps national du Viêt-nam dont les eaux fraîches ruisselantes des montagnes nourrissent les rizières: "Je retrouve dans cette image de terre et d'eau le Viêt-nam de mon enfance," dit Kim Lefèvre, tout en reconnaissant qu'en partant, elle "ne laisser[a] plus de trace dans ce paysage d'eau où il recommence à pleuvoir" (221-22). Ne parlons pas des "rêveries" qu'apporteraient les eaux selon la thèse de Gaston Bachelard ni de la "mécanique" féministe des liquides selon Luce Irigaray, la thématique de l'eau dans la littérature et le cinéma vietnamiens semble désigner un lien profond et intime entre le corps et le "lieu" vietnamiens (il ne s'agit évidemment pas des poncifs occidentaux de terre et de sol). Condensée par Nguyên Du dans la scène célèbre du Truyên Kiêu, ce texte fondamental à la culture du Viêt-nam, l'image du corps féminin dans son extase aquatique dépasse toute captation simplement voyeuriste ou vulgairement lubrique pour nous tracer la voie "sans traces" du chemin fluvial qui remonte vers l'âme profonde (comme on dit "pays profond") du dât nuòc. II s'agit donc d'un topos à lire dans sa spécificité culturelle, au delà de toute idéale de "sang et sol", vers une expression on ne peut plus lyrique d'identité localisée, une affirmation du chez soi collectif et donc d'une résistance limpide mais inlassable à toute imposition de l'extérieur. 


\section{Bibliographie}

ANH Hung, Tran (1993), L'odeur de la papaye verte / Mùi du du Xanh. France/ Viet Nam, 104 minutes.

BACHELARD, Gaston (1942), L'eau et les rêves. Paris: José Corti.

Du, Nguyên (1802/1961). Truyên Kiêu / Kim-Vân-Kiêu. Traduction Xuân Phuc et Xuân Viêt, Paris: Gallimard/Unesco.

IRIGARAY, Luce (1977), Ce sexe qui n'en est pas un. Paris: Minuit.

LeFEVRE, Kim (1989). Métisse blanche. Paris: Bernard Barrault.

LefEVRE, Kim (1995). Retour à la saison des pluies. Paris: Aube.

MINH-HA, Trinh T. (1995). A Tale of Love / Une histoire d'amour. USA, 108 minutes.

MiNH-HA, Trinh T. (1999). Cinema Interval. New York: Routledge, 1999.

VERLAINE, Paul (1874): Romances sans paroles. Paris: Léon Vanier. 
